\title{
Participation in a Science Festival Promotes Inclusive Science Communication around Autism Spectrum Disorder
}

1 Chris Gunter ${ }^{1,2^{*},+}$, Cynthia B Sinha ${ }^{3}$, David Jaquess ${ }^{1,++}$

$2{ }^{1}$ Marcus Autism Center, Children's Healthcare of Atlanta and Department of Pediatrics, Emory

3 University School of Medicine, Atlanta, GA, USA

$4{ }^{2}$ Department of Human Genetics, Emory University School of Medicine, Atlanta, GA, USA

$5{ }^{3}$ Division of Pediatric Hematology-Oncology-BMT, Emory University School of Medicine, Atlanta, GA, 6 USA

$7{ }^{+}$Current address: National Human Genome Research Institute, National Institutes of Health,

8 Bethesda, MD, USA

$9 \quad{ }^{++}$Current address: HERE! Psychological Services, Atlanta, GA, USA

10

$11 *$ Correspondence:

12 Chris Gunter

13 chris.gunter@nih.gov

14 ORCID 0000-0001-9369-7537

15 Keywords: autism, autism spectrum disorder, science communication, science festival, inclusive 16 science communication, inductive content analysis 


\section{Abstract}

18 As a high prevalence disorder with limited information about etiology, autism spectrum disorder (ASD) has been marked by confusion and miscommunication around its causes and treatments. To promote high-quality science communication, we participated in a local science festival, both providing information about the brain and ASD and asking passersby questions about their knowledge of ASD. We then asked the booth staffers to evaluate the program and conducted qualitative analyses of public and staffer responses. Public responses to the question "what would you like to know about autism?" most often concerned how the disorder was diagnosed or defined. In contrast, public responses to the question "what would you like others to know about autism?" centered around educating those unaffected by ASD on how to improve interactions and awareness, mentioning inclusivity and intersectionality, and dispelling negative stereotypes. The staffers overwhelmingly reported that in future years, they would include even more science and allow for more in-depth conversations with interested parties, as well as bringing materials in other languages. These responses are in keeping with a trend for more inclusive science communication, particularly in the field of behavioral health and ASD, and a desire to challenge myths around the condition. We conclude that our science festival interactions brought multiple benefits to public and 33 staff.

\section{Introduction}

Autism spectrum disorder, as defined in the Diagnostic and Statistical Manual of Mental Disorder, Fifth Edition is a neurodevelopmental disorder with key features reflecting impairment in social interactions (e.g., reciprocal communication and social interaction) and difficulties arising from overly circumscribed or repetitive interests, activities and/or behaviors (1). These disorders are present from very early in life, and they range in a spectrum of severity from mild (reflected in persistent difficulties with perspective taking and comprehending affective components of human relations) to very severe (complete withdrawal from social interaction and lack of awareness of socially mediated features of the environment). The recent epidemiological estimate of prevalence from the Centers for Disease Control and Prevention (CDC) are 1 in 54 children overall, with the rate being four times higher in males than in females (2). Age of diagnosis is crucial to receiving early treatment and affecting the trajectory of the condition; given the behavioral nature of ASD, symptoms do change over the lifespan and as a result some percentage of children may move above and below diagnostic thresholds over time (3).

In studying the apparent physical underpinnings of ASD, the scientific community has struggled to provide a complete explanation of etiology. Although some estimates point to genetics to account for up to $80 \%$ of phenotypic variance (4), the full developmental dynamic for ASD remains to be discerned. This combination of a high prevalence disorder with limited information about etiology creates a dynamic in which misinformation about etiology and treatment of the condition is rampant. While searches for the word "autism" on the internet yield hundreds of millions of hits, the quality of information varies widely and can influence, for example, the opinions of parents on accepting a diagnosis (5) or health policy makers setting standards for coverage of autism treatment (6). Healthcare consumers (as well as providers) often struggle to sort through the overwhelming volume of information to land on an empirically-informed vantage point $(6,7)$. In sum, these are 
which there are effective assessments and empirically supported interventions that offer promising outcomes.

61 Public awareness, understanding, and discussion of ASD has changed substantially in the last 20

62 years, in part due to the sharp rise in number of diagnoses (8) and in part due to the rise in

63 pseudoscience such as rumored links between vaccines and ASD (9). Given the 1 in 58 reported

64 national prevalence for children meeting diagnostic criteria (10), and the increasing depiction of ASD in the media, it is very likely that most people in the US know someone or know of someone with ASD. Self-advocates have played an important role in changing the conversation as well, by describing their experiences and advocating for a shift from "autism awareness" to "autism acceptance," for example (11). An emerging view in the advocate community is to shift from labeling ASD a "disorder" and instead to view it as part of a spectrum or even a circular rainbow of diverse brain functioning $(12,13)$. This is in keeping with drives for inclusion and recognition of diversity in other communities.

Research suggests that these disorders are present across demographic characteristics; however, they are detected at differing rates across gender, race ethnicity, and SES. In the US there are differences in the age of diagnosis and access to care, related to race, ethnicity and socioeconomic status, as well as intersectional concerns $(10,14,15)$. Further, people with developmental disorders like ASD tend to have reduced access to healthcare and health information, as do ethnic and racial minorities and those from lower income households (16). Thus, individuals on the autism spectrum who also come from an underserved demographic group have been particularly poorly served in the healthcare system. Given the emerging evidence base demonstrating the importance of early diagnosis and intervention in ASD (17), there is a clear need for clear and accurate communication around ASD, consistently available to everyone from specialists to the families at their moment of expressing concerns about their child's development.

This additional element in the landscape of ASD research and communication is a dynamic of racial disparity in access to care and related delayed diagnosis and intervention is consistent with the concept of intersectionality in diversity-differences within differences that make a difference (18). Within any movement for equality and social justice, it is not uncommon for there to be blind spots to the differences within a recognized group. As a result, individuals whose identities fall at the intersection of classes seem to encounter doubly disparate access to care. To address these intersections, networks of self-advocates living on the autism spectrum have created alliances with disability-rights groups, LGBTQ alliances, and other movements for social justice and representation (19).

As the view of ASD has changed over time, so has the need for providers and scientists to communicate findings and recommendations around ASD. For example, erroneous reports of vaccine-autism links have taken up significant energy and sowed confusion around causes of ASD, although sites such as Vaccines Today are now providing high quality online communication and vaccine information (20). Smaller qualitative studies (21) have described a journey of progressive engagement with science for the parents of children diagnosed with ASD, whether to seek out explanations to help them understand the condition or to seek appropriate/better services for their children. A larger study in the US (22) comparing beliefs and understanding of parents and scientists regarding ASD reported that there was significant discordance between parents and scientists on their beliefs about causes of ASD and research priorities. In partial contrast, a large European study (23) found that the autism community surveyed was generally supportive of autism research, 
103

104

105

106

107

108

109

110

111

112

113

114

115

116

117

118

119

120

121

122

123

124

125

126

127

128

129

130

131

132

133

134

135

136

137

138

139

140

141

142

143

recommending that community perspectives be continually surveyed and considered in the design of research studies, including the use of preferred terminology such as "at-risk" infants. We believe that successful communication with families seeking information about ASD must include interactive and inclusive messages and activities, welcoming multiple perspectives and tailoring itself to formal or informal settings, and therefore designed our public engagement activity accordingly. This may involve intentional inclusion of words or other documentation of views from families that include a person with ASD, such as families from racial or ethnic backgrounds other than the dominant culture (e.g., Latinx, African-American, recent immigrant, east-Asian, Indian); families including parents and caregivers of many family structures; and people living on the autism spectrum of differing genders, ages, and/or races. Luisi, Rodgers and Schultz (24) argued that science communication requires training opportunities, which should include experiential learning, and they pointed out that this type of training begs a framework for program evaluation.

Given our desire to communicate high-quality scientific information about ASD, we included participation in multiple public events in the Dissemination and Outreach Core for our NIH-Funded Autism Center of Excellence grant (NIMH 2P50 MH100029). Specifically, we sought to engage with members of the public at a public science event, the Atlanta Science Festival, which closes with an "Exploration Expo" attracting up to 30,000 people. Science festivals are becoming a more common method for interaction with the public, including in areas of controversial science (25), and can be a valuable tool for evaluating public engagement with scientific topics. Our booth, entitled "How Does Your Brain Talk?", offered both scientific information and a chance for passersby to answer specific questions about possible knowledge gaps regarding ASD. The booth also included a visual exemplar of brain development in the form of an animated portrayal of brain development research findings. Such exemplars have been found to increase the impact of science communications about autism to the general public (26). The booth was staffed by science trainees and junior faculty members who had been communicating with the public for months in the context of tours of Marcus Autism Center and were provided a one-hour seminar on how to approach the public, how to anticipate and respond to "hot topics" that might reflect misinformation, and how to communicate key pieces of information pertinent to the booth. To assess the experiential training aspect of working in the booth, we asked questions of the individuals staffing the booth about their experience and its anticipated impact on future science communication activities. As noted by Patton (27), a qualitative analysis using a theme analysis is a common first step in program evaluation. We used this type of analysis to evaluate the feedback from both groups of stakeholders: the attendees and the booth staffers.

\section{Statement of Ethical Review}

The authors reviewed the purpose, design, execution and use of this project and determined that it fit the definition of Program Evaluation as stipulated by Emory University (http://www.irb.emory.edu/forms/review/programeval.html). The Emory University IRB's NonHuman Subjects Research Determination Electronic Form agreed that this project was exempt from review by the university's Institutional Review Board.

\section{Methods}

Terminology 
144 In the larger community related to autism spectrum disorders, we see the terms autism and ASD both used frequently. We also see "people with autism/ASD" and "autistic people" used and would generally defer to the choice of the person involved. In this article, we will use all four of these terms.

Booth setup

Our booth at the Atlanta Science Festival in March 2019 included a mock magnetic resonance imaging (MRI) scanner, constructed from cardboard and a table, with sound effects for how an MRI might sound through headphones. As part of the booth experience, attendees were able to lay on the table and have the staffers, primarily students or faculty from the Marcus Autism Center, move cardboard parts of the mock MRI above them while playing the sound effects and explaining how an MRI takes pictures of the brain. [No research or evaluation were conducted or collected on this completely voluntary part of the festival booth.] Afterwards (or separately if they chose not to try the mock scanner), they would be shown a rotating brain image on a monitor, depicting the wiring of circuits in the brain as found through neuroimaging studies at our Center. At the front of the booth were flip charts as described below.

Data Collection

Data were collected to evaluate two aspects of the community outreach event. The first question of what type of information would fit the needs of the audience at the event was evaluated by asking two questions.

163 Question 1: "What would you like to know about autism?"

Question 2: "What would you like others to know about autism?"

These questions were posted atop two 20 -inch by 23 -inch flip charts in the display booth and on the wall of the booth. Staffers directed attendees' attention to the questions as they began interacting with the display. Two corresponding methods were used to gather responses for both questions. One consisted of the staffers in the booth transcribing oral responses from attendees onto the flip charts where the questions were posted or asking the attendees to write their own response onto the flip chart. The second method was to provide small self-adhesive note pads on which attendees wrote their responses and posted them on the wall of the booth. We did not collect any information about attendees and cannot match them to their comments. Following the event, responses gather in both methods were transcribed into a single text file.

The second research question was how the staffers experienced the role of communicating with the public and what (if anything) they learned. In order to gather this information, staffers were asked to complete a questionnaire of four open-ended questions.

1. What was the main thing you learned from this experience?

2. How did you change what you were communicating over the course of the day?

3. What did you learn about people's perception about autism or science in general? coding was not able to match respondent to response. 
185 Responses from the event were analyzed using content analysis approach. This analytic approach

186 facilitated the conceptual organization of the attendee responses (28). Content analysis utilizes

187 deductive or inductive coding. The former coding technique purposefully codes for established set

188 of words, phrases, or concepts (29). Inductive coding develops concepts from the responses without

189 establishing codes a priori (29), and therefore was used for this evaluation. Once conceptual coding

190 was completed, patterns were identified, and the concepts were grouped into meaningful

191 categories. Frequently, content analysis involves quantifying coding categories and may retain only

192 the most prevalent (29). For this evaluation, all responses were retained for the final analysis. The

193 lead coder (CBS) developed the coding concepts and initial categories. For inter-coder reliability, a

194 second and third coder reviewed coding concepts and edited organization of the categories for

195 clarity. Consensus was reached for the final analysis. Given our small data set, we did not believe

196 utilizing a statistical measure for reliability was appropriate; our aim was $100 \%$ agreement.

197 As an additional means of analyzing themes in the responses from attendees, we analyzed the text

198 of the public responses using the Semantic Word Clouds Visualization tool $(30,31)$. We produced two separate visualizations: first (Figures 1A and 2A), we applied a simple layout of sorting by rank. Number of words was set at 50 . Similarity was determined by the cosine coefficient, ranking was by term frequency, sizing was 4:3, and color was black. Second (Figures 1B and 2B), we used the layout settings for the seam carving algorithm, which determines an image based on semantic relationships and then minimizes the number of empty spaces between groups of words (31). Number of words was set at 100 . Similarity was also determined by the cosine coefficient, ranking was by term frequency, sizing was 4:3, and color was ColorBrewer 2. In all figures, stop words and numbers were removed, similar words were grouped, and the shortest word was set at three 207 letters.

Results

Approximately 150 attendees engaged with the booth during the event; 27 responses were collected for Question 1 and 30 were collected for Question 2. The median length of response was eight and nine words for Question 1 and Question 2 respectively. Attendees appeared to include diversity in ages, gender, ethnic origin, and relationship to ASD; in discussion with the booth staffers, attendees reported themselves to be from multiple professions and family situations. Many passersby saw the name of the booth sponsor (Marcus Autism Center) and stopped by specifically to tell us about their family member, student, patient, or friend with ASD. A few individuals specifically self-identified as having ASD. Therefore, it is clear that from the general audience who self-selected by attending a science festival, our evaluation participants represent a subset with a much higher likelihood of interest in or personal relationship to ASD science. themes. Overall, for Question 1, people appeared to be most interested in learning how ASD is diagnosed or defined. There were also multiple questions about causes for ASD, including environmental factors (but not including vaccines). Finally, specific treatment questions were raised, including service locations in the area, transitioning from childhood to adulthood and beyond, and diet or counseling requests.

For Question 2, the responses centered around educating those unaffected by ASD on how to 226 improve interactions and awareness and dispelling negative stereotypes. Specific advice on how to 227 treat them in the emergency room, for example, was mixed in with general advice such as "They 
want to interact but it's harder for them!" Many of the statements mentioned positive characteristics like creativity, intelligence, and even "the most sweet and thoughtful people you will have the pleasure of knowing." To combat negative labels, attendees mentioned specifically "it's not a disease" and "autism does not define a person." We also saw comments that seemed to reflect an intersectionality of marginalized experiences, e.g. "Black autistic folk exist too," and multiple reminders of inclusivity, e.g. "It is not a disease, just a different way of thinking." These responses appeared to have been provided by attendees who were on the autism spectrum or those that had experienced autism in their families, offering support for a subjective experience of intersectional barriers to societal inclusivity or to access of medical care. questions suggested additional thematic trends. Figures 1 and 2 represent these responses in word clouds, using different analysis methods.

\section{autism diagnosed problems communicate people start brains ASD change age parent} diagnosis pregnancy son children nervous happened distinguish solutions verbally adulthood benefits tools services transitions Atlanta daughter disabilities denial treatment role affect kids detect families common diet behaviors pesticides childhood MRI learning sensory

A. differences microbiome stage risk related time typically

B.
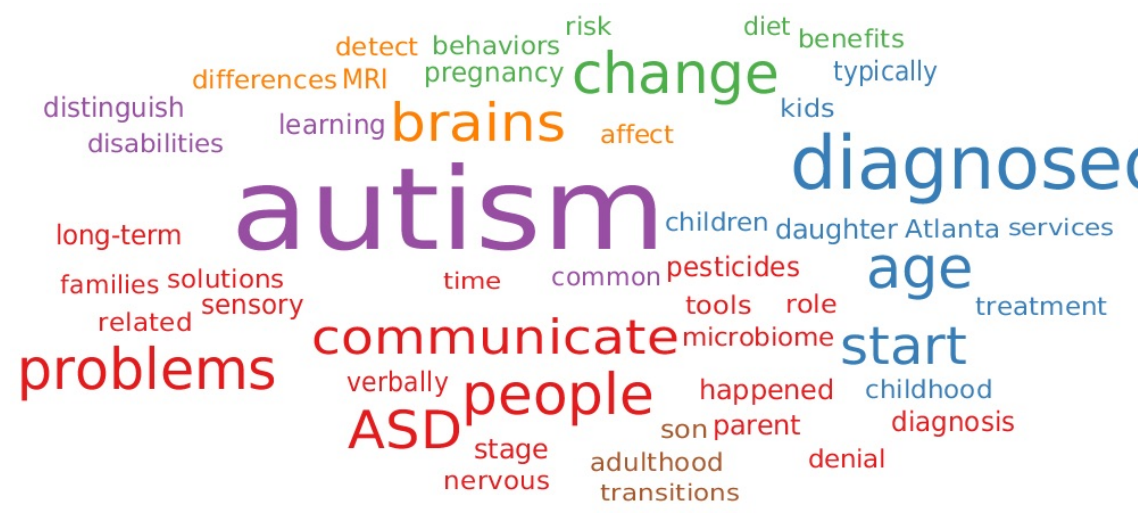

Figure 1: Textual analysis of answers to the question, "What do you want to know about autism?" 
B.

A. define

sweetest

\section{autism}

smart things give disease exist disorder personally Kids wearing interact autistic awesome

sensitivities refuse strengths thoughtful good approach radiology

friends favorite intellectually levels work general loyal weighted difficult

misunderstand texture lights creative folk necessarily disabled love educated terminology lonely apron proper lot interested patient eat

Figure 2: Textual analysis of answers to the question, "What do you want others to know about autism?" A: Answers sorted by rank and term frequency. B. Answers sorted by seam carving algorithm and term frequency.

Figures $1 \mathrm{~A}$ and $2 \mathrm{~A}$ depict the top 50 words in rank order, with size of the word corresponding to its frequency in the answers. Figures $1 \mathrm{~B}$ and $2 \mathrm{~B}$ depict the top 100 words in a semantic word cloud, placing related words near each other and sizing each word based on its frequency in the answers. For Question 1 about what attendees would like to know, the common themes primarily centered around defining and describing the disorder (diagnosis, brain, problems, nervous, detect, problems, start) with a clear thread of words humanizing the condition (children, kids, people, age, older [individuals]; Figure 1). With regards to Question 2, the common themes include issues of increased awareness qualities of individuals with autism (many, look, smart, different, kids, Black), challenges related to the condition (sensitivities, eat, wear, trying, interact), and areas of dialogue on how to characterize the condition (disease, disorder, different; Figure 2).

265 All seven of the staffers responded with extensive information on the program evaluation questionnaire, with a median response length of 63 words per question, overall. The comments from the staffers reflected some of those from the attendees, including motivation to learn more 
about ASD themselves, and to bring more awareness about ASD to the community. A number expressed surprise at the intense level of interest in the science around ASD (and the enthusiasm of children for trying out the mock MRI scanner), but confirmed that as scientists, they enjoyed translating their scientific work and learning about the concerns and questions of those interested in ASD but not working in a scientific setting. They reported changing their approach for science communication based on the individual, sometimes focusing just on the brain and other times more on ASD.

The staffers also made specific suggestions about the booth layout: more space was needed because it became crowded at peak times, and a more sophisticated MRI scanner would be optimal. There was some disagreement at the Festival about whether it is best to reach out to people walking by instead of waiting for them to approach the booth, but in their responses, multiple staffers said the next booth should feature more engaging activities or visuals, and the team should plan to engage all passersby, perhaps with a short script. In keeping with an inclusive approach, one staffer specifically asked for some items in Spanish to be created for the next time, because "I had a few families come up to me who only spoke Spanish. I was able to translate for them, but they wanted materials to take home with them and I would've loved to have provided them with materials in Spanish." Clearly, the prominent desire for next year was to include even more science and allow for more in-depth conversations with interested parties.

\section{Discussion}

287 From the responses to our Question 1, "What would you like to know about autism," the responses most often included questions on how ASD is diagnosed or defined. This result is likely to be affected by the theme of the booth, as it is related to a larger project to detect and diagnose ASD at earlier ages, and therefore this theme was much more likely to be mentioned by the staffers interacting with those entering the booth and interacting with them. In addition, we saw multiple questions about the specifics of ASD symptoms and manifestations, whether behavioral, cognitive, or social. These themes are likely influenced by the personal relationships many of our generalpublic attendees disclosed, including as a teacher or nurse, for example. Family members of people with autism were more likely to ask specific treatment queries, such as "Where can older children get services in Atlanta, my daughter was diagnosed at age 13."

Overall, the responses we received when we asked what people wanted to know about ASD reflect larger questions in the community as a whole, based on our experiences interacting with the community for multiple years as a researcher (CG) and clinician (DJ). How can we help children as they transition into adulthood? What does scientific research tell us about ASD symptoms, diagnoses, and treatments? What are the specific symptoms of ASD, and what do terms like "highfunctioning" mean? And what is new research, such as into the microbiome or the role of pesticides, telling us about ASD?

We did not record any questions about vaccines, and none were written on the flip-chart boards or Post-It notes. This suggests that either attendees asked those questions directly to booth staff (and would have been told that there is no link between vaccines and autism), and/or that the public who stopped by did not have this question or did not want to put it into writing.

308 For Question 2, on what others should know about autism, we saw many more references to 309 inclusivity and the more personal side of the condition. Overwhelmingly, respondents want to teach 310 those who are unaffected on appropriate ways to interact with individuals with ASD. This included 
311 advice on patience and not "taking things personally," as well as specifics like "think twice before

312 wearing cologne/perfume" due to sensory sensitivities. Respondents wanted to share the positive

313 attributes of people they knew with ASD and counter the idea that autism and intellectual disability

314 are conflated (four comments specifically mentioned that people with autism are "smart" or that

315 they "aren't necessarily intellectually disabled!").

316 Individuality of people with ASD was a prominent theme as well, echoing themes we have heard

317 from self-advocates for years like "Not all kids with autism look the same." Simultaneously, there

318 was also a theme of request for inclusion, such as "We exist too," "We're not that different," and

319 "Many people with autism are lonely....Can be good friends!"

320 Finally, we heard about the intersectionality of ASD with the experience of being a minority, from comments like "Black autistic folk exist too." We grouped this into a theme with requests for "seeing" the autistic person, including other statements like "Just because they don't 'look' autistic doesn't mean they aren't."

324 We conclude from our content analyses that the science festival brought multiple benefits to our efforts to communicate with the public. At the same time, there is clearly value to the ASD community in seeing themselves represented at such an event. Some self-identified autistic individuals stayed for in-depth conversations and shared their experiences with us. Our staffers reported they enjoyed learning from those with ASD too, and that they would use the experience to shape future research and future interactions. In addition, their responses did seem to reflect growth in science communication through expanded knowledge of public need and increased repertoire for engaging with them. The greater variety of hands-on activities in the booth suggested by staffers could function as multiple visual exemplars and thus support increased learning for attendees (26). In future festival booths, we would also like to recruit some staffers or volunteers who identify as being autistic themselves.

The primary limitation of this work is the difficulty in generalization from our findings, given its nature as an evaluation of a one-time event. This evaluation may also provide guidance for future research in this area. Data collection could be expanded either by repeating the same event as we had planned to do (unfortunately, the 2020 Atlanta Science Festival was canceled due to the coronavirus pandemic) or repeating this setup at another festival to increase the sample size. These added events would allow a more refined analysis of responses and possible exploration of issues related to intersectionality. Researchers might need to gather data from multiple events or in multiple locations within a science fair to achieve this goal. Based on the themes derived from the present evaluation, future research could formalize a set of information to be communicated to meet the needs identified here; such studies should include a measure of efficacy in getting the message across to members of the public. It also could be informative to include different methods of communication (perhaps including different language translations) within a single event in order to compare the efficacy of those methods. In addition, designing a study that gathered contact information would allow researchers to follow-up and gather data about the durability of information communicated to attendees. Each of these directions could further expand our understanding of effective science communication.

\section{Conflict of Interest}

352 The authors declare that the research was conducted in the absence of any commercial or financial 353 relationships that could be construed as a potential conflict of interest. 


\section{Author Contributions}

CG and DJ designed the evaluation and the questionnaires. CS completed the first round of coding, and all authors participated in later iterations of analysis. All authors contributed to manuscript revision, read and approved the submitted version.

\section{Funding}

This evaluation was funded by Emory University and Marcus Autism Center. CG and DJ were funded in part by NIMH grant 2P50 MH100029, with additional support from the Marcus Foundation and Whitehead Foundation.

\section{Acknowledgments}

We thank all of the attendees at our Atlanta Science Festival exhibit, the Festival organizers, and the volunteers at the exhibit: Aiden Ford, Skai Glasser, Emma Goldman, Cheryl Klaiman, Longchuan Li, Sarah Markert, Adriana Mendez, Jack Olmstead, Tristan Ponzo, and Sarah Shultz. CG also thanks the Fondation Brocher.

References

1. American Psychiatric Association. American Psychiatric Association: Diagnostic and Statistical Manual of Mental Disorders Fifth Edition. Arlington. 2013.

2. Maenner MJ, Shaw KA, Baio J, Washington A, Patrick M, DiRienzo M, et al. Prevalence of autism spectrum disorder among children aged 8 Years-Autism and developmental disabilities monitoring network, 11 Sites, United States, 2016. MMWR Surveill Summ. 2020;69(4):1-12.

3. Ozonoff S, Young GS, Landa RJ, Brian J, Bryson S, Charman T, et al. Diagnostic stability in young children at risk for autism spectrum disorder: a baby siblings research consortium study. J Child Psychol Psychiatry. 2015;n/a-n/a.

4. Bai D, Yip BHK, Windham GC, Sourander A, Francis R, Yoffe R, et al. Association of Genetic and Environmental Factors With Autism in a 5-Country Cohort. JAMA Psychiatry. 2019 Jul 17;1-9.

5. Pham T, Kuznetsova A, Gim H, Cordrey K, Milanaik R. The Internet's Effect on Parental Trust in Pediatrician Diagnosis of Autism and Likelihood of Seeking a Second Opinion. J Autism Dev Disord. 2019;49(10):4355-62.

6. Keenan M, Dillenburger K. How 'Fake News' Affects Autism Policy. Societies. 2018 May 9;8(2):29.

7. Paynter J, Luskin-Saxby S, Keen D, Fordyce K, Frost G, Imms C, et al. Evaluation of a template for countering misinformation-Real-world Autism treatment myth debunking. Webster AA, editor. PLoS One. 2019 Jan 30;14(1):e0210746.

8. Hansen SN, Schendel DE, Parner ET. Explaining the Increase in the Prevalence of Autism Spectrum Disorders. JAMA Pediatr. 2015 Jan 1;169(1):56.

9. Jain A, Marshall J, Buikema A, Bancroft T, Kelly JP, Newschaffer CJ. Autism Occurrence by MMR Vaccine Status Among US Children With Older Siblings With and Without Autism. JAMA. 2015 Apr 21;313(15):1534. 
394

395

396

397

398

399

400

401

402

403

404

405

406

407

408

409

410

411

412

413

414

415

416

417

418

419

420

421

422

423

424

425

426

427

428

429

430

431

432

433

434

435

436

437

438

439

10. Baio J, Wiggins L, Christensen DL, Maenner MJ, Daniels J, Warren Z, et al. Prevalence of Autism Spectrum Disorder Among Children Aged 8 Years - Autism and Developmental Disabilities Monitoring Network, 11 Sites, United States, 2014. MMWR Surveill Summ. 2018 Apr 27;67(6):1-23.

11. Des Roches Rosa S. Autism Acceptance Day \& Month: Do This, Not That [Internet]. THINKING PERSON'S GUIDE TO AUTISM. [cited 2020 Jul 28]. Available from: http://www.thinkingautismguide.com/2017/04/autism-acceptance-day-month-do-thisnot.html

12. Gunter C. Seeing the spectrum entire. Nature. 2015 Aug 20;524(7565):288-9.

13. Silberman S. Neurotribes: The Legacy of Autism and How to Think Smarter About People Who Think Differently. Allen \& Unwin. 2015.

14. Travers JC, Tincani M, Krezmien MP. A Multiyear National Profile of Racial Disparity in Autism Identification. J Spec Educ. 2013 May 29;47(1):41-9.

15. Singh JS, Bunyak G. Autism Disparities: A Systematic Review and Meta-Ethnography of Qualitative Research. Qual Health Res. 2018;104973231880824.

16. Benevides TW, Carretta HJ, Lane SJ. Unmet Need for Therapy Among Children with Autism Spectrum Disorder: Results from the 2005-2006 and 2009-2010 National Survey of Children with Special Health Care Needs. Matern Child Health J. 2016;20(4):878-88.

17. Zwaigenbaum L, Penner M. Autism spectrum disorder: advances in diagnosis and evaluation. BMJ. 2018 May 21;361:k1674.

18. Perlman M. The origin of the term 'intersectionality' [Internet]. Columbia Journalism Review. 2018 [cited $2020 \mathrm{Jul} 28$ ]. Available from:

https://www.cjr.org/language_corner/intersectionality.php

19. Nerenberg J. Does Neurodiversity Have a Future? [Internet]. Greater Good. 2016 [cited 2020 Jul 28]. Available from:

https://greatergood.berkeley.edu/article/item/does_neurodiversity_have_a_future

20. Finnegan G, Holt D, English PM, Glismann S, Thomson A, Salisbury DM, et al. Lessons from an online vaccine communication project. Vaccine. 2018 Oct;36(44):6509-11.

21. Feinstein NW. Making sense of autism: Progressive engagement with science among parents of young, recently diagnosed autistic children. Public Underst Sci. 2014 Jul 5;23(5):592-609.

22. Fischbach RL, Harris MJ, Ballan MS, Fischbach GD, Link BG. Is there concordance in attitudes and beliefs between parents and scientists about autism spectrum disorder? Autism. 2016 Apr 26;20(3):353-63.

23. Fletcher-Watson S, Apicella F, Auyeung B, Beranova S, Bonnet-Brilhault F, Canal-Bedia R, et al. Attitudes of the autism community to early autism research. Autism. 2016 Mar 14;

24. Luisi MLR, Rodgers S, Schultz JC. Experientially learning how to communicate science effectively: A case study on decoding science. J Res Sci Teach. 2019;56(8).

25. Rose KM, Korzekwa K, Brossard D, Scheufele DA, Heisler L. Engaging the Public at a Science Festival. Sci Commun. 2017 Apr 25;39(2):250-77.

26. Dixon GN, Mckeever BW, Holton AE, Clarke C, Eosco G. The Power of a Picture: Overcoming Scientific Misinformation by Communicating Weight-of-Evidence Information with Visual Exemplars. J Commun. 2015;65(4):639-59.

27. Patton MQ. Qualitative Research \& Evaluation Methods: Integrating Theory and Practice. 3rd ed. Thousand Oaks, California: SAGE Publications; 2014.

28. Vaismoradi $\mathrm{M}$, Turunen $\mathrm{H}$, Bondas $\mathrm{T}$. Content analysis and thematic analysis: Implications for conducting a qualitative descriptive study. Nurs Health Sci. 2013 Sep;15(3):398-405. 
bioRxiv preprint doi: https://doi.org/10.1101/2021.01.04.425324; this version posted January 5, 2021. The copyright holder for this preprint (which was not certified by peer review) is the author/funder. This article is a US Government work. It is not subject to copyright under 17 USC 105 and is also made available for use under a CCO license.

440 29. Elo S, Kyngäs H. The qualitative content analysis process. J Adv Nurs. 2008;62(1):107-15. 441 30. Semantic Word Cloud Visualization - Description [Internet]. [cited 2020 Jul 28]. Available from: http://wordcloud.cs.arizona.edu/description.html

31. Barth L, Kobourov SG, Pupyrev S. Experimental comparison of semantic word clouds. In: Lecture Notes in Computer Science (including subseries Lecture Notes in Artificial Intelligence and Lecture Notes in Bioinformatics). 2014. 